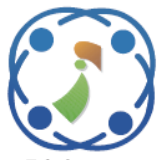

\title{
Know Sure Thing based Machine Learning Strategy for Predicting Stock Trading Signals
}

\author{
Arjun Singh Saud ${ }^{1 *}$ \\ Subarna Shakya ${ }^{2}$ \\ ${ }^{I}$ Central Department of Computer Science and Information Technology, Tribhuvan University, Kathmandu, Nepal \\ ${ }^{2}$ Department of Electronics and Computer Engineering, IOE, Tribhuvan University, Kathmandu, Nepal \\ * Corresponding author’s Email: arjunsaud@cdcsit.edu.np
}

\begin{abstract}
Although some studies employed technical indicators as input to machine learning models to forecast stock trading signals, there was a noticeable gap between how technical analysts and machine learning professionals used technical indicators. Based on this finding, this study suggested a know sure thing based machine learning (KST-ML) technique for anticipating stock trading signals and compared its results to KST-based trading and BuyHold strategies. The main idea behind the KST-ML strategy is to learn and then predict stock trading signals from the relationship between KST indicators such that more accurate predictions can be made. The strategies were assessed based on their annual rate of return (ARR), Sharpe ratio (SR), and percentage of profitable trades executed. In addition, the proposed strategy's performance was compared to that of several intelligent stock trading strategies proposed in the literature. The proposed method clearly outperformed the other two strategies in terms of all three evaluation measures. With a 5.6 SR value, the strategy generated a $67.73 \%$ ARR and made 79.38\% profitable trades. The results were much better than the results from the KST-based and the Buy-Hold strategies. Furthermore, we found that the annual returns generated by the KST-ML approach were significantly higher than those generated by other intelligent techniques reported in the literature.
\end{abstract}

Keywords: Intelligent stock trading, KST indicator, KST-ML, Stock prediction, Stock trading signal.

\section{Introduction}

The primary market and secondary market are the two types of stock markets. The primary market is the stock market where public firms sell stocks to the general public via an initial public offering (IPO) or a follow-on public offering (FPO). Secondary market is the stock market where stock traders meet, negotiate, and trade stocks at the current prevailing market price. Stock traders always try to predict future direction of stocks and trade accordingly. Fundamental analysis and technical analysis are two schools of thought to forecast the direction of future stock prices [1]. Fundamental analysis focuses on overall economic, industry, and company analysis to predict future stock price movement, whereas technical analysis focuses on price action, which provides insight into supply-demand dynamics and aids in predicting future stock price movement.
Technical analysts analyze past trading data to predict stock price direction [2-4]. Therefore, machine learning strategies to stock prediction are closely related with prediction strategies used by technical analysts.

Technical indicators and candlestick chart analysis are widely adopted by technical analysts and machine learning researchers to forecast stock price movement [5-8]. Technical indicators are computed from the historical price and volume of stock trading. Moving average convergence divergence (MACD) indicator, know sure thing (KST) oscillator, average movement directional indicator (ADMI), relative strength index (RSI), moving average (MA), momentum, bollinger band (BB), William \%R, etc. are widely used technical indicators in the technical analysis community [9]. Although some machine learning models only used historical trading data to forecast financial markets [10-12], historical trading data along with technical 
indicators also being used to improve accuracy of financial market forecasting [13-16].

KST oscillator is a popular technical indicator among technical analysts to predict stock buy/sell signals. Although many machine learning researchers used this indicator as an input feature to the machine learning model to predict stock price movement [17-19], there was gap between the way of using this indicator by the machine learning experts and technical analysts. Therefore, this research work proposed a KST oscillator based machine learning strategy (KST-ML) to predict buy/sell signals. The proposed strategy integrated machine learning approach with the approach used by technical analysts. The KST-ML strategy is devised on the basis of KST-based trading strategy used by technical analysts. Basics of the trading strategy is introduced in section 2. They use relationship between KST indicators to predict stock trading signals. However, the biggest issue with the KST oscillator is that it frequently generates misleading trading signals and is also a lagging indicator. The main objective of the proposed strategy is to learn true patterns so that buy/sell signals can be generated in timely way and misleading trading signals can be filtered out. This study also evaluated the performance of the proposed strategy against the KST-based trading strategy and the Buy-Hold strategy. Furthermore, the study compared the results obtained from the KST-ML strategy with the various intelligent trading strategies found in the literature. The annual rate of return (ARR), Sharpe ratio (SR), and proportion of profitable trades executed by the strategies were used to assess their performance.

Although there were numerous intelligent trading methods devised in the literature [20], none of them was based on concepts used by technical analysts for forecasting stock trading signals. Another motive of the proposed KST-ML method is to demonstrate that more profitable intelligent trading strategies can be developed based on the theories that technical analysts use to forecast financial markets.

Time series modelling approaches like autoregressive integrated moving average (ARIMA) [20-21], and machine learning strategies like logistic regression (LR) [22-23], support vector machine (SVM) [24-25], random forest (RF) [26-28], decision trees [27-28], etc. are widely used by researchers to predict stock market. Besides, artificial neural network approaches like multilayer perceptron (MLP) are also used by many researchers to forecast financial markets [29-31]. The aforementioned strategies lack ability to remember the context in contrast to recurrent neural networks (RNNs). Meanwhile, due to the challenges of exploding/vanishing gradient descent associated with RNNs, its variations such as the long shortterm memory (LSTM) network [32-38] and the gated recurrent unit (GRU) network [37-40] became state-of-the-art models for stock forecasting and other time series prediction problems. Therefore, the GRU network was employed as a machine learning tool in this study to forecast stock trading signals. The major reason behind using GRU network is its capability of generalizing in better way with moderate volume of data compared to LSTM network. Furthermore, GRU networks have a simpler structure than LSTM networks, allowing them to be trained faster.

The rest of the paper is organized as follows. Section 2 discusses the concept of the KST oscillator briefly. The proposed KST-ML strategy is illustrated in section 3. The methodology adopted to carry out the research work is described in section 4. Experimental results and discussions are provided in section 5. Finally, section 6 concludes the research outcome.

\section{Know sure thing (KST) oscillator}

The KST oscillator is a trend and momentum oscillator. It is calculated from rate of change (ROC) indicators. The ROC indicator is a momentum indicator that measures the difference in percentage between a stock's current price and its price $\mathrm{N}$ periods ago. KST oscillator is merely the sum of simple moving averages (SMAs) of the ROCs of four different periods, and KST signal is simply the 9-period SMA of the KST oscillator. Eq. (1) gives the mathematical formulation for computing the oscillator. This oscillator is widely used for determining the stock market's primary swings. Buying stocks when the KST line crosses above the KST signal line and selling the stocks when the KST line crosses below the KST signal line is the standard KST indicator based swing trading strategy. In this article, this strategy is referred as KST-based trading strategy. If the KST lines continue above the zero line, the momentum is in favour of bulls, and if the KST lines stays below the zero line, the momentum is in favour of bears [9, 7-19].

$$
\begin{aligned}
& \text { ROC\#1 }=\left(C P_{t}-C P_{t-10}\right) / C P_{t-10} \\
& \text { ROC\#2 }=\left(C P_{t}-C P_{t-15}\right) / C P_{t-15} \\
& \text { ROC\#3 }=\left(C P_{t}-C P_{t-20}\right) / C P_{t-20} \\
& \text { ROC\#4 }=\left(C P_{t}-C P_{t-30}\right) / C P_{t-30}
\end{aligned}
$$




$$
\begin{aligned}
& K S T=S M A_{10}(R O C \# 1) \times 1+S M A_{10}(R O C \# 2) \\
& \times 2+S M A_{10}(R O C \# 3) \times 3+S M A_{15}(R O C \# 4) \times 4
\end{aligned}
$$

Where, $\operatorname{SMA}_{\mathrm{p}}(x)$ is p-period SMA of $x$ and $\mathrm{CP}_{\mathrm{t}}$ is close price of $\mathrm{t}^{\text {th }}$ trading day.

\section{Proposed strategy}

This section describes the proposed target feature generation model used for producing buy/hold/sell signals, KST-ML strategy, and trading simulation model used for performing automated stock trading based on predicted signals.

\subsection{Target feature generation}

On the basis of crossover between the KST indicator line and the KST signal line, this study devised a model for producing target feature "Action". Eq. (2) is the mathematical formulation of the model. The model alternates between producing buy and sell signals. When the indicator line and the signal line intersect, the model puts three buy or sell signals in the target feature. The main motivation behind this is to make it easier for machine learning model to spot trade signals.

$$
\begin{array}{ll}
\text { If } K[i]>K S[i] & \text { action }[i: i+3]={ }^{\prime} \text { Buy }^{\prime} \\
\text { If } K[i]<K S[i] & \text { action }[i: i+3]={ }^{\prime} \text { Sell' } \\
\text { Otherwise } & \text { action }[i]=' \text { 'Hold' }^{\prime}
\end{array}
$$

Where $\mathrm{K}[\mathrm{i}]$ and $\mathrm{KS}[\mathrm{i}]$ are KST indicator and KST signal of $i^{\text {th }}$ trading day.

\subsection{KST-ML strategy}

The conceptual framework of the proposed KST-based machine learning (KST-ML) strategy for predicting stock trading signals is presented in Fig. 1. Calculation of KST indicators is discussed in section 2. The target feature generation model is presented in section 3.1. Scaling, encoding and data preparation strategies used in the research study are discussed in sections 4.2 and 4.3. The gated recurrent unit (GRU) network used for predicting stock trading signals is described in section 4.4. Finally, the trading simulation module performs automated trading on the basis of predicted signals as discussed in section 3.3.

\subsection{Trading simulation}

This research work also designed and constructed a trading simulator that takes trading

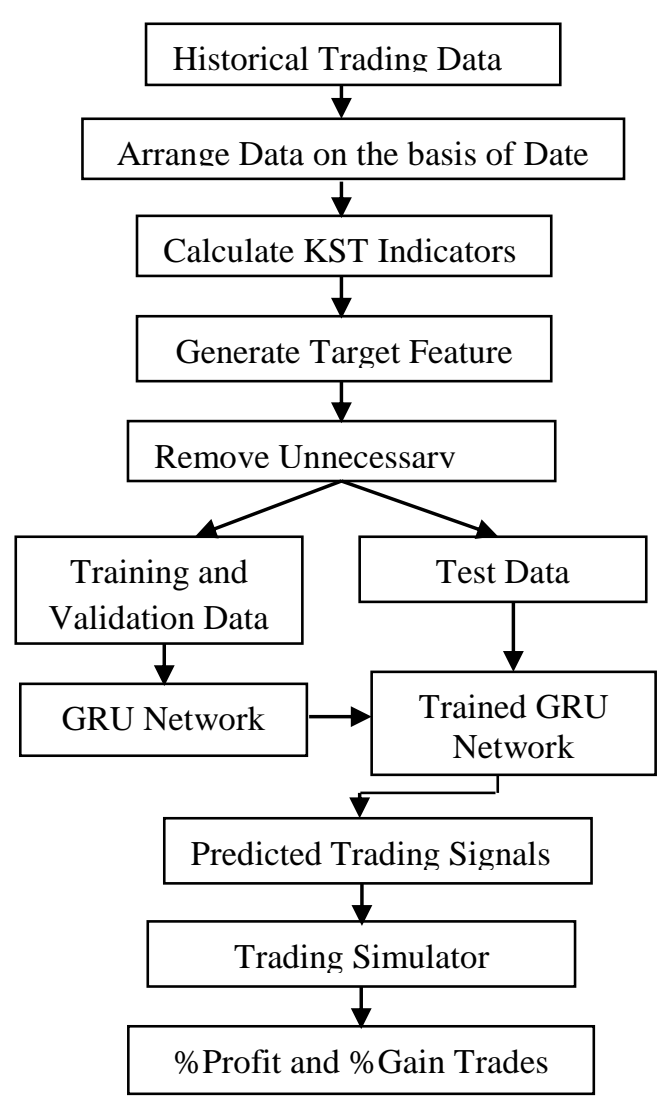

Figure. 1 Proposed KST-ML strategy

signals predicted by the KST-ML strategy or KSTbased trading strategy and performs automated stock trading. The simulator buys and sells stocks based on the assumption that stock investors have no stocks at the start. Therefore, the simulator's first trading operation should be a buy operation. As a result, the simulator executes a series of buy and sell operations. The sequence consists of the equal number of both operations if the last transaction is a sell operation. However, if the last transaction is a buy operation, the sequence will include one more buy action than the number of sell actions. In this situation, the simulator discards the last buy operation and calculates profit/loss derived from automated trading. The "close price" is assumed to be the buy/sell price by the simulator. The simulator assumes that the investor has some seed money to invest in equities. It reinvests the entire sold value in the following buy transaction after the stocks are sold. Eq. (3) gives formula for calculating the gross profit/loss earned from trading. The cost of transactions and the tax on capital gains are not factored into the equation. Finally, the simulator uses Eq. (4) to compute profit/loss percentage.

$$
p l=y-x
$$


Table 1. Historical trading data of stocks

\begin{tabular}{|c|c|c|c|}
\hline \multirow{2}{*}{ Stock Exchange } & \multirow{2}{*}{ Stock Name } & \multicolumn{2}{|l|}{ Date } \\
\hline & & From & To \\
\hline \multirow{5}{*}{ NEPSE } & Standard Chartered Bank Nepal (SCBN) & $4 / 15 / 2010$ & $7 / 01 / 2021$ \\
\hline & Nepal SBI Bank Ltd.(NSBI) & $4 / 15 / 2010$ & $7 / 01 / 2021$ \\
\hline & Muktinath Bikas Bank Ltd. (MNBBL) & $11 / 3 / 2011$ & $7 / 01 / 2021$ \\
\hline & Prime Life Insurance (PLIC) & $7 / 22 / 2010$ & $7 / 01 / 2021$ \\
\hline & Chilime Hydropower Company Ltd. (CHCL) & $4 / 15 / 2010$ & $7 / 01 / 2021$ \\
\hline \multirow{5}{*}{ BSE } & Housing Development Finance Corp. Ltd. (HDFC) & $1 / 1 / 2000$ & $7 / 01 / 2021$ \\
\hline & Larsen \& Toubro Ltd. (LT) & $1 / 1 / 2000$ & $7 / 01 / 2021$ \\
\hline & Hindustan Zinc Ltd. (HZ) & $1 / 1 / 2000$ & $7 / 01 / 2021$ \\
\hline & JSW Steels Ltd. (JSW) & $1 / 1 / 2000$ & $7 / 01 / 2021$ \\
\hline & Vedanta Ltd. (VL) & $1 / 1 / 2000$ & $7 / 01 / 2021$ \\
\hline \multirow{5}{*}{ NYSE } & Baker Hughes Company (BKR) & $1 / 1 / 2000$ & $7 / 01 / 2021$ \\
\hline & Carnival Corporation \& plc. (CCL) & $1 / 1 / 2000$ & $7 / 01 / 2021$ \\
\hline & Devon Energy Corporation (DVN) & $1 / 1 / 2000$ & $7 / 01 / 2021$ \\
\hline & The Kroger Co. (KR) & $1 / 1 / 2000$ & $7 / 01 / 2021$ \\
\hline & Nokia Corporation (NOK) & $1 / 1 / 2000$ & $7 / 01 / 2021$ \\
\hline \multirow{5}{*}{ SSE } & GuangYuYuan Chinese Herbal Medicine (GCHM) & $1 / 1 / 2000$ & $7 / 01 / 2021$ \\
\hline & Hengtong Optic-Electric (HOE) & $8 / 22 / 2003$ & $7 / 01 / 2021$ \\
\hline & Hua Xia Bank Limited (HB) & $6 / 19 / 2003$ & $7 / 01 / 2021$ \\
\hline & Shanxi Coking Ltd. (SCC) & $1 / 1 / 2000$ & $7 / 01 / 2021$ \\
\hline & Orient Group Incorporation (OG) & $1 / 1 / 2000$ & $7 / 01 / 2021$ \\
\hline
\end{tabular}

Where, $\mathrm{y}$ is the amount of money obtained from last sell operation and $x$ is amount of seed money.

$$
p l p=p l / x \times 100
$$

\section{Methodology}

This section describes dataset, data preprocessing and preparation strategy, configuration of GRU network, and performance measures used in this research work.

\subsection{Dataset}

Historical stock trading data from four stock exchanges was used in this study: the Shanghai stock exchange (SSE), the New York stock exchange (NYSE), the Bombay stock exchange (BSE), and the Nepal stock exchange (NEPSE). Twenty stocks were experimented, five from each stock market. Table 1 gives description the stock data in detail. The start date of the data was varied due to data availability. The data of NEPSE and BSE stocks were obtained from the Nepal stock exchange [41] and the BSE India [42], respectively. Similarly, Yahoo finance [43] was used to obtain data on NYSE and SSE stocks.

\subsection{Data preprocessing}

Initially, the historical trading data was organized in chronological order from the oldest to the most recent date. The KST indicators were then generated, including the KST oscillator, KST signal, and KST histogram, and any superfluous features were removed from the dataset. Following that, the proposed target generation model in Eq. (2) was used to generate the target attribute 'Action'. Finally, standard scalar was used to normalize the input features, and one-hot encoding technique was used to encode the target feature.

\subsection{Data preparation}

The datasets were partitioned into train, validation, and test sets in an 8:1:1 ratio. The goal of this research work was to anticipate stock trading signals for $(t+1)^{t h}$ day utilizing input features from day $(t+N-1)$ to day $t$, where $t$ is current trading day and $N$ is window size. Thus, the study's input data included a combination of $N$ independent variables $d_{t-N+1}, \ldots . d_{t-1}, d_{t}$ and a dependent variable $a_{t}$, where $d_{i}$ is a tuple $\left(k_{i}, k s_{i}, k h_{i}\right)$ and $a_{i}$ reflects transaction activity for the $i^{\text {th }}$ trading day. The symbols $k_{i}, k s_{i}$ and $k h_{i}$ represents KST oscillator, KST signal, and KST histogram, respectively. The size of window $(\mathrm{N})$ employed in the research study was 5 as suggested by Saud and Shakya [44].

\subsection{Configuration of GRU network}

The GRU network employed in this study had a configuration of $3 \times 100 \times 100 \times 3$. A dropout layer with a dropout rate of 0.2 was placed after 
each hidden layer of the network. The network's hidden layers employed the ReLU activation function, whereas the output layer used the Softmax activation function. With the GRU network, the Adam gradient descent optimizer was also used. This configuration of the GRU network is not guaranteed to be optimal; nonetheless, experiments were undertaken with many configurations before settling on this one.

\subsection{Performance measures}

The annual rate of return (ARR), Sharpe ratio (SR), and proportion of profit/loss transactions conducted by trading strategies were all used to analyze trading strategies in this study. SR measures performance of the stock trading against risk-free asset. Higher value of SR means higher excess return compared to risk-free asset. ARR and SR were computed from the profit earned from the trading strategies using the formulae given in Eqs. (5) and (6) respectively. Since the target feature 'Action' is simply an estimation based on the crossover between KST line and KST signal line, classification accuracy is not a relevant performance measure for the proposed strategy.

$$
A R R=\left\{(1+r)^{1 / n}-1\right\} \times 100
$$

Where, $\mathrm{n}$ is the number of years and $\mathrm{r}$ is return from stock trading.

$$
S R=\left(R_{t}-R_{f}\right) / \sigma
$$

Where, Rt is the return from stock trading, $\mathrm{Rf}$ is risk-free return, and $\sigma$ is standard deviation of Rt.

\section{Results and discussion}

As already mentioned, this research work experimented 20 stocks. Price movement pattern of these stocks in the test period is shown in Fig. 2. From the figure we can see that the stocks SCBN, NSBI \& NOK are in sideways pattern, stocks MNBBL, CHCL, PLIC, JSW, VL, KR \& GCHM are in bullish pattern, stocks CCL, HOE \& HB are in bearish pattern and stocks HDFC, LT, HZ, BKR, DVN, SCC \& OG are in mixed pattern during the test period. In the figure $\mathrm{x}$-axis represents number of trading days and y-axis represents price. Fig. 3 shows buy/sell operations for the stocks SCBN, HDFC, BKR, and HOE that were carried out using a trading simulator based on forecasted signals by the KST-ML approach.

\subsection{Analysis of returns}

The trading simulator executed buy/sell operations on the test data on the basis of predicted signals and computed returns obtained from stock
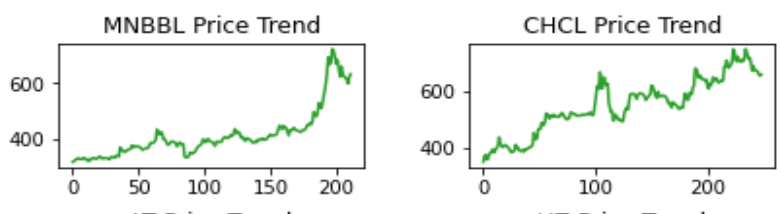

LT Price Trend
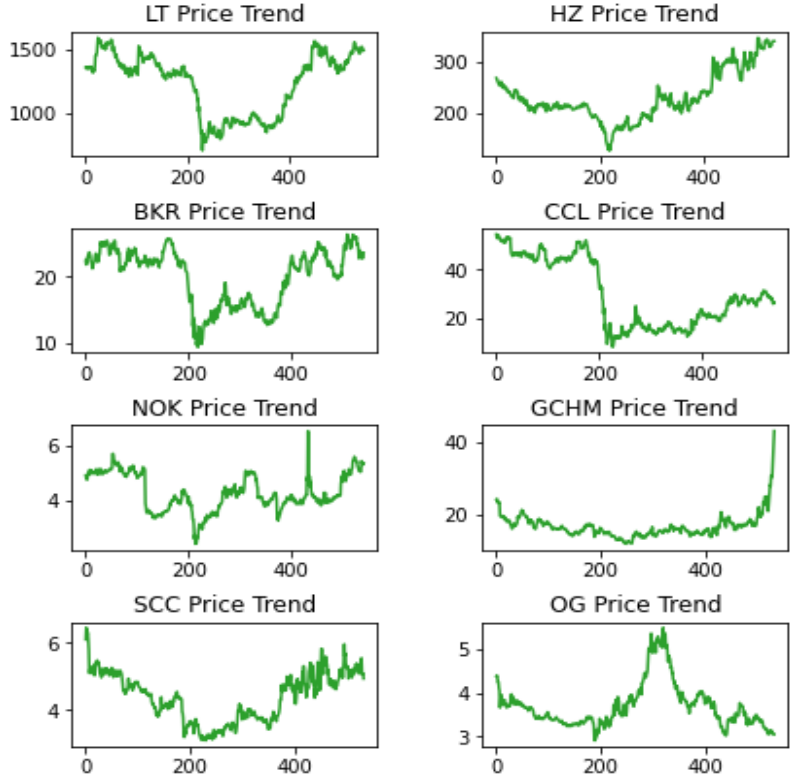

Figure. 2 Price trend of stocks in test period 

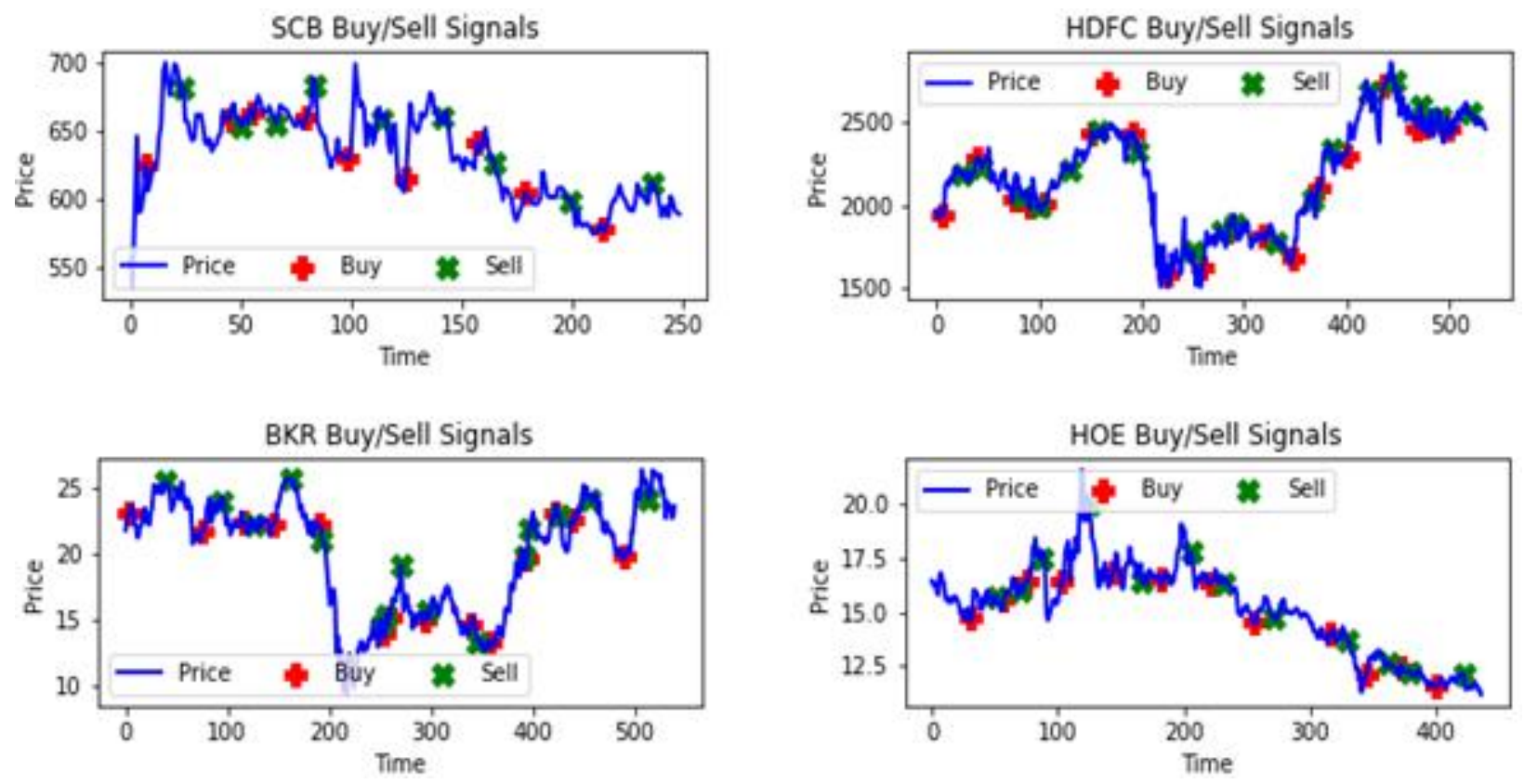

Figure. 3 Buy/Sell signals of stocks

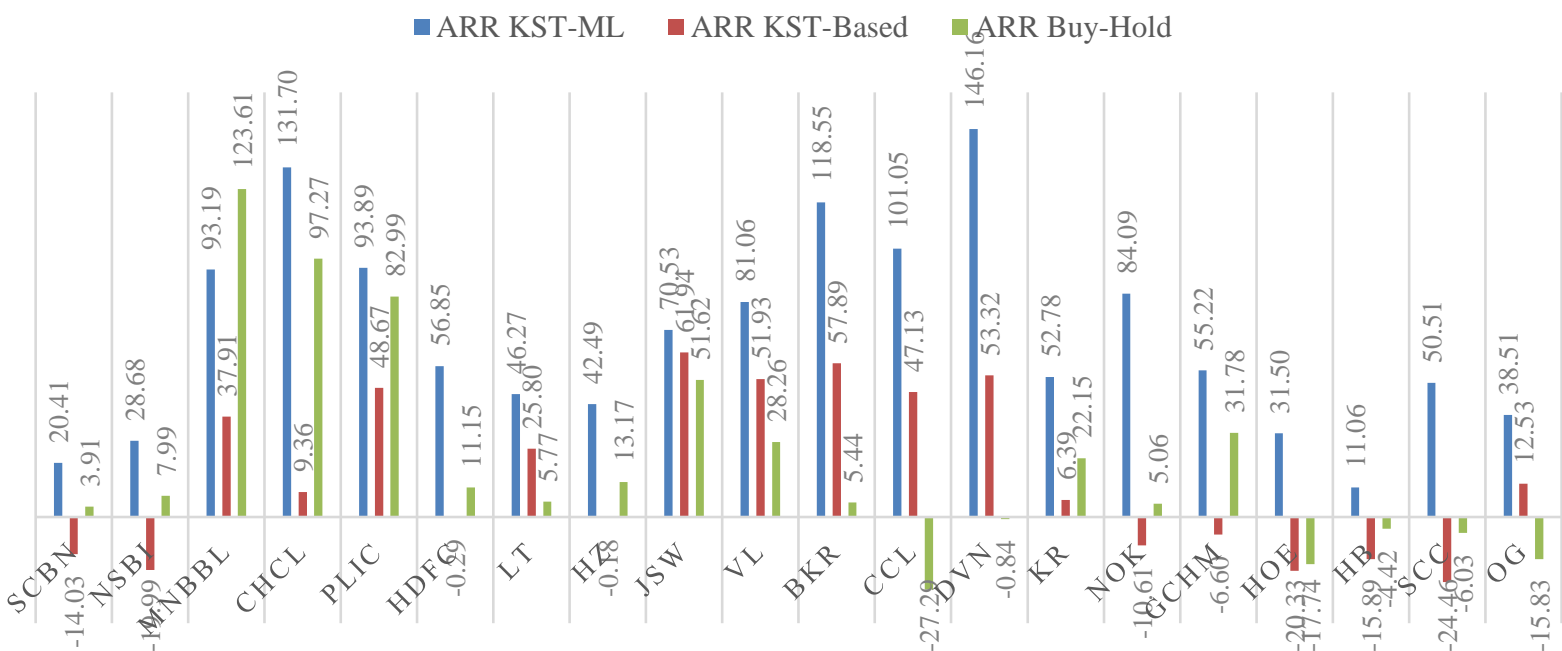

Figure. 4 ARR obtained from trading strategies

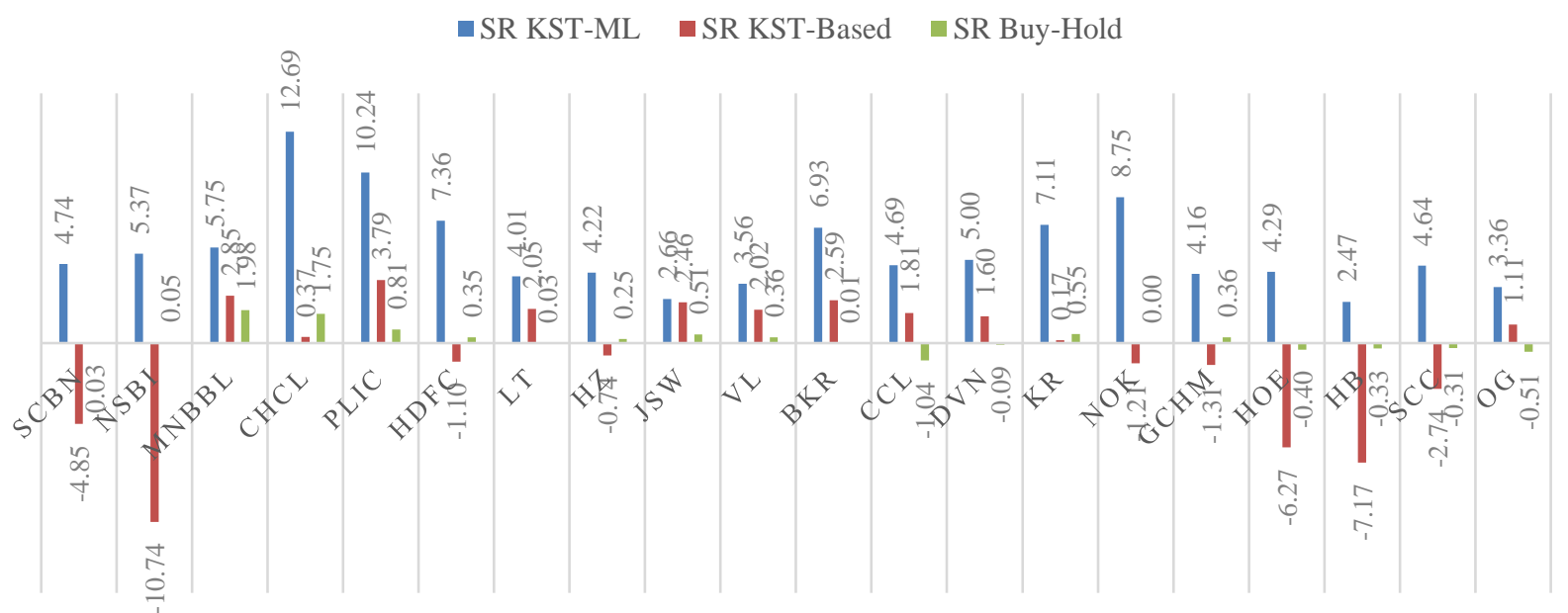

Figure. 5 SR obtained from trading strategies 


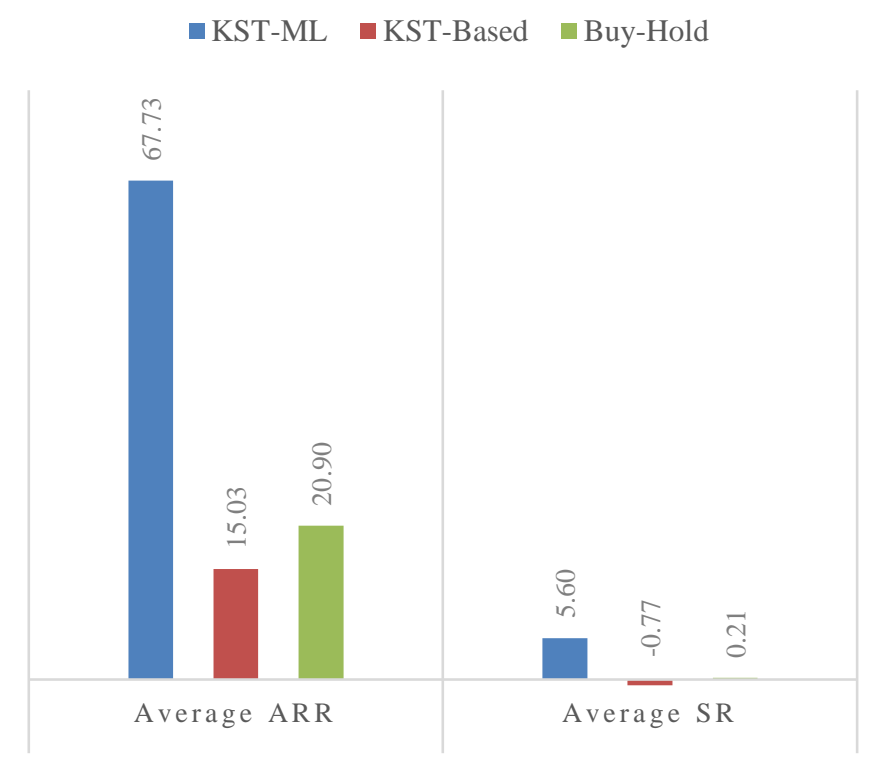

Figure. 6 Average ARR and SR

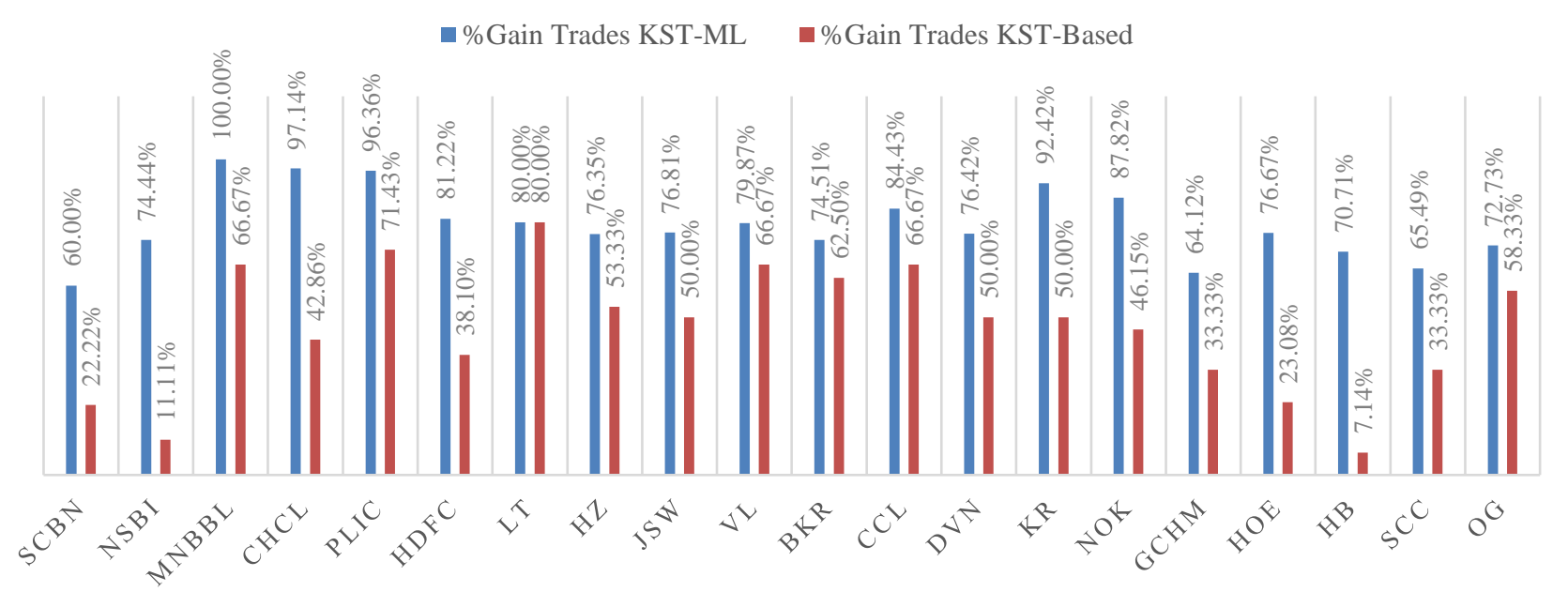

Figure. 7 Percentage gain trades

trading. Thereafter, ARR was calculated using Eq. (5) and the result is presented in Fig. 4. In order to quantify the risk associated with stock trading, SR was then calculated using Eq. (6) and the result is presented in Fig. 5. Treasury bill rates from the respective countries were used as risk-free returns in the SR calculation. Furthermore, the standard deviation of trading returns was utilized in the KSTML and KST-based strategies, and the standard deviation of the past 10 years annual returns was employed in the Buy-Hold strategy.

The KST-ML trading approach clearly outperformed the KST-based trading strategy and the Buy-Hold strategy in terms of ARR and SR, as shown in Figs. 4 and 5. The strategy yielded $11.06 \%$ to $146.16 \%$ ARR from stock trading. This
ARR was achieved with SR range of 2.47 to 12.69 . The Buy-Hold strategy achieved ARR from $-27.29 \%$ to $123.61 \%$ with SR value ranged from -1.09 to 1.98 . The KST-based trading strategy performed worst and yielded ARR from $-24.46 \%$ to $61.94 \%$ with SR range -10.74 to 2.59 . In summary, the KST-ML strategy yielded $67.73 \%$ average ARR with 5.6 average SR value. Meanwhile, the BuyHold and the KST-based strategy achieved average ARR $20.9 \%$ and $15.03 \%$ with SR values 0.21 and -0.77 respectively as shown in Fig. 6. From the observation of ARR, we saw that the Buy-Hold strategy obtained satisfactory returns only from bullish stocks. The KST-based method exhibited unpredictable behaviour. With certain stocks, the technique produced satisfactory returns, while with 
others, it produced disappointing returns. However, the KST-ML strategy achieved satisfactory returns from all stocks regardless of the stock trend.

From Fig. 5, we also observed that the KST-ML strategy achieved SR above 3 for 18 stocks and below 3 only for 2 stocks. However, SR values obtained from the Buy-Hold strategy and the KSTbased strategy was always below 3. Furthermore, the Buy-Hold strategy achieved positive SR for 13 stocks and negative SR for remaining 7 stocks and the KST-based strategy achieved positive SR for 11 stocks and negative SR for remaining 9 stocks. Based on these observations, it is clear that the KSTML approach is the most profitable and safest trading method of the three.

\subsection{Analysis of gain trades}

The percentage of gain trades executed by trading strategies is one of the important measure. The trading simulator counts total number of trades and total number of profitable trades executed by the trading strategies. Then, percentage of profitable trades executed by the strategies were calculated and the result is presented in Fig. 7. The Buy-Hold strategy is not analyzed in this regard because it only conducts one trade per stock during the test period.

The percentage of profitable trades conducted by the KST-ML trading strategy is substantially higher than the percentage of such trades done by the KST-based strategy, as shown in Fig. 7. The KST-ML strategy executed $60 \%$ to $100 \%$ profitable trades whereas the KST-based strategy executed only $7.14 \%$ to $80 \%$ such trades. On average, the trading strategies executed $79.38 \%$ and $46.65 \%$ profitable trades respectively. This observation leads to the fact that a considerable percentage of trade signals generated by the KST indicator were misleading signals that were significantly filtered by the KST-ML approach.

\subsection{Comparison with other intelligent trading strategies}

Stoen, Paja, and Sandita proposed HC-LSTM and HC-CNN strategies for stock price prediction along with trading simulation based on heuristicbased approach and found that the HC-LSTM strategy performed better than the HC-CNN strategy. The HC-LSTM strategy was able to achieve $10.61 \%$ more return than the Buy-Hold strategy \& $14.17 \%$ more return than the bollinger band based trading strategy [35]. Yang, Hao, Cai, Chen, and Ren predicted stock trading signal based on multiindicator channel convolutional neural networks
(MICNN) and found that the MICNN strategy achieved $6.2 \%$ more return than the RSI based trading strategy [45]. Kroha and Friedrich compared genetic algorithms for trading strategies and found that the genetic programming based trading strategy achieved $2.72 \%$ more return than the Buy-Hold strategy [46]. Song and Lee predicted stock price movement with LSTM neural networks and reported $81.6 \%$ accuracy and profit near to zero [47]. Matsubara, Akita, and Uehara predicted stock price by deep neural generative model of news articles and obtained accuracy above $60 \%$ and $22 \%$ loss [48]. Vargas, Anjos, Bichara and Evsukoff used deep learning for stock market prediction using technical indicators and financial news articles and obtained $10.72 \%$ more profit than the than the BuyHold strategy [49]. Tan and Wang proposed SDNN for optimized trading strategy that was able to generate $33.3 \%$ annualized return [50]. Sezer and Ozbayoglu utilized 2-D deep CNN and technical indicator to develop algorithmic trading system that generated $5.85 \%$ more annualized return than the Buy-Hold strategy [51]. Oncharoen and Vateekul added risk-reward function into loss function to train deep neural network and reported $21.56 \%$ annualized return [52]. From this survey, we found that most of intelligent trading strategies compared performance with Buy-Hold strategy and related technical indicator based trading strategy. Thus, in this study, the proposed KST-ML approach has been compared to the Buy-Hold strategy and the KSTbased trading strategy.

As presented in section 5.1, we observed that the proposed KST-ML strategy yielded $52.7 \%$ and $46.82 \%$ more return than the KST-based and BuyHold strategy respectively. The results are far superior to those produced using the aforementioned intelligent trading strategies. The strength of the KST indicator, on which the KST-ML strategy was designed, is the main reason for the proposed approach's improved performance. Technical experts have been using this indicator for a long time. Its strength, on the other hand, is not exploited in any intelligent stock trading method. From these discoveries, we claim that the proposed KST-ML strategy not only outperformed the Buy-Hold and KST-based strategies but also excelled other intelligent trading methods proposed in the literature in terms of annualized return.

\section{Conclusion}

This paper proposed a know sure thing based machine learning (KST-ML) strategy for predicting buy/hold/sell signals in stock trading and compared 
the model's performance to that of a Buy-Hold strategy and a KST-based trading strategy. The annualized rate of return (ARR), Sharpe ratio (SR), and percentage of gain trades executed were used to analyze the strategies.

Experimental results revealed the fact that the KST-ML strategy performed better than the other two strategies in terms of all three evaluation measures. The approach's average ARR was 52.7 percent and 46.82 percent greater than the average ARR of the KST-based trading strategy and the Buy-Hold strategy, respectively. The KST-ML strategy yielded the 5.6 average SR from the trading which was only -0.77 and 0.21 for the other two strategies. This finding demonstrated that the KSTML strategy is both more profitable and less risky than the other two trading methods. The strategy was able to generate satisfactory returns from the stock trading regardless of the stock trend. The KST-ML method, on the other hand, averaged 79.38 percent profitable trades, while the KST-based approach averaged only 46.65 percent profitable trades. This discovery leads to the fact that the KSTML approach may effectively filter out a large number of false trade signals generated by the KST indicator. Furthermore, we found that the KST-ML approach generated significantly greater annual returns than other intelligent trading techniques reported in the literature. Thus, we conclude that the proposed KST-ML method is superior to other trading strategies for automatic stock trading. This research conclusion has a significant implication: it is possible to develop intelligent trading strategies based on correlations between technical indicators that have long been used by technical analysts.

\section{Conflicts of interest}

The authors declare no conflict of interest.

\section{Author contributions}

Arjun Singh Saud played a key role in framing conceptual design, implementation, result analysis, and research report writing. Subarna Shakya played the role of advisor in this research work. He provided feedback, validated the result and reviewed the research report.

\section{Acknowledgments}

This research work was supported by the University Grant Commission, Nepal under Grant No. PhD-75/76-S\&T-9.

\section{References}

[1] E. Ahmadi, M. Jasemi, L. Monplaisir, M. A. Nabavi, A. Mahmoodi, and P. Amini Jam, "New efficient hybrid candlestick technical analysis model for stock market timing on the basis of the Support Vector Machine and Heuristic Algorithms of Imperialist Competition and Genetic", Expert Syst. Appl., Vol. 94, pp. 21-31, 2018.

[2] J. Singh and M. Khushi, "Feature Learning for Stock Price Prediction Shows a Significant Role of Analyst Rating", Appl. Syst. Innov., Vol. 4, No. 1, p. 17, 2021.

[3] I. K. Nti, A. F. Adekoya, and B. A. Weyori, "A systematic review of fundamental and technical analysis of stock market predictions", Artif. Intell. Rev., Vol. 53, No. 4, pp. 3007-3057, 2020.

[4] T. Anbalagan and S. U. Maheswari, "Classification and Prediction of Stock Market Index Based on Fuzzy Metagraph", Procedia Comput. Sci., Vol. 47, pp. 214-221, 2015.

[5] K. Prachyachuwong and P. Vateekul, "Stock Trend Prediction Using Deep Learning Approach on Technical Indicator and Industrial Specific Information", Information, Vol. 12, No. 6, p. 250, 2021.

[6] F. B. Oriani and G. P. Coelho, "Evaluating the impact of technical indicators on stock forecasting", In: Proc. of IEEE Symposium Series on Computational Intelligence (SSCI), Athens, Greece, pp. 1-8, 2016.

[7] C. C. Hung and Y. Chen, "DPP: Deep predictor for price movement from candlestick charts", PLoS One, Vol. 16, No. 6, p. e0252404, 2021.

[8] Y. Udagawa, "Predicting Stock Price Trend Using Candlestick Chart Blending Technique", In: Proc. of IEEE International Conference on Big Data, Seattle, WA, USA, pp. 4709-4715, 2018.

[9] R. W. Colby, The Encyclopedia of Technical Market Indicators, $2^{\text {nd }}$ edition. New York, NY, USA: McGraw-Hill Education, 2002.

[10] M. Roondiwala, H. Patel, and S. Varma, "Predicting Stock Prices Using LSTM", Int. J. Sci. Res., Vol. 6, No. 4, pp. 1754-1756, 2017.

[11] L. D. Persio and O. Honchar, "Recurrent neural networks approach to the financial forecast of Google assets", Int. J. Math. Comput. Simul., Vol. 11, pp. 7-13, 2017.

[12] B. Yang, Z. Gong, and W. Yang, "Stock market index prediction using deep neural network ensemble", In: Proc. of 36th Chinese Control 
Conference (CCC), Dalian, China, pp. 38823887, 2017.

[13] G. Zhang, X. Zhang, and H. Feng, "Forecasting financial time series using a methodology based on autoregressive integrated moving average and Taylor expansion", Expert Syst., Vol. 33, No. 5, pp. 501-516, 2016.

[14] R. Arévalo, J. García, F. Guijarro, and A. Peris, "A dynamic trading rule based on filtered flag pattern recognition for stock market price forecasting", Expert Syst. Appl., Vol. 81, pp. 177-192, 2017.

[15] M. Qiu and Y. Song, "Predicting the Direction of Stock Market Index Movement Using an Optimized Artificial Neural Network Model", PLoS One, Vol. 11, No. 5, p. e0155133, 2016.

[16] R. Dash and P. K. Dash, "A hybrid stock trading framework integrating technical analysis with machine learning techniques", J. Financ. Data Sci., Vol. 2, No. 1, pp. 42-57, 2016.

[17] D. Oyewola, E. G. Dada, O. E. Olaoluwa, and K. A. A. Mustapha, "Predicting Nigerian Stock Returns using Technical Analysis and Machine Learning", Eur. J. Electr. Eng. Comput. Sci., Vol. 3, No. 2, pp. 1-8, 2019.

[18] Y. Peng, P. H. M. Albuquerque, H. Kimura, and C. A. P. B. Saavedra, "Feature selection and deep neural networks for stock price direction forecasting using technical analysis indicators", Mach. Learn. with Appl., Vol. 5, p. 100060, 2021.

[19] A. S. Saud and S. Shakya, "3-Way Gated Recurrent Unit Network Architecture For Stock Price Prediction", Indian J. Comput. Sci. Eng., Vol. 12, No. 2, pp. 421-427, 2021.

[20] A. W. Li and G. S. Bastos, "Stock Market Forecasting Using Deep Learning and Technical Analysis: A Systematic Review", IEEE Access, Vol. 8, pp. 185232-185242, 2020.

[21] A. A. Ariyo, A. O. Adewumi, and C. K. Ayo, "Stock Price Prediction Using the ARIMA Model", In: Proc. of 16th International Conference on Computer Modelling and Simulation, Cambridge, UK, pp. 106-112, 2014.

[22] J. Gong and S. Sun, "A New Approach of Stock Price Prediction Based on Logistic Regression Model”, In: Proc. of International Conference on New Trends in Information and Service Science, Beijing, China, pp. 1366-1371, 2009.

[23] S. Syed, M. Mubeen, A. Hussain, and I. Lal, "Prediction of stock performance by using logistic regression model: evidence from
Pakistan Stock Exchange (PSX)", Asian $J$. Empir. Res., Vol. 8, No. 7, pp. 247-258, 2018.

[24] Z. Hu, J. Zhu, and K. Tse, "Stocks market prediction using Support Vector Machine", In: Proc. of $6^{\text {th }}$ International Conference on Information Management, Innovation Management and Industrial Engineering, Xi'an, China, pp. 115-118, 2013.

[25] B. M. Henrique, V. A. Sobreiro, and H. Kimura, "Stock price prediction using support vector regression on daily and up to the minute prices", J. Financ. Data Sci., Vol. 4, No. 3, pp. 183-201, 2018.

[26] I. K. Nti, A. F. Adekoya, and B. A. Weyori, "A comprehensive evaluation of ensemble learning for stock-market prediction”, J. Big Data, Vol. 7, No. 1, p. 20, 2020.

[27] S. R. Polamuri, K. Srinivas, and A. K. Mohan, "Stock Market Prices Prediction using Random Forest and Extra Tree Regression", Int. J. Recent Technol. Eng., Vol. 8, No. 3, pp. 12241228, 2019.

[28] M. Obthong, N. Tantisantiwong, W. Jeamwatthanachai, and G. Wills, "A Survey on Machine Learning for Stock Price Prediction: Algorithms and Techniques", In: Proc. of the $2^{\text {nd }}$ International Conference on Finance, Economics, Management and IT Business, Prague, Czechia, pp. 63-71, 2020.

[29] A. V. Devadoss and T. A. A. Ligori, "Forecasting of Stock Prices Using Multi-Layer Perceptron”, Int. J. Web Technol., Vol. 2, No. 2, pp. 52-58, 2013.

[30] P. Gao, R. Zhang, and X. Yang, "The Application of Stock Index Price Prediction with Neural Network", Math. Comput. Appl., Vol. 25, No. 3, p. 53, 2020.

[31] M. P. Naeini, H. Taremian, and H. B. Hashemi, "Stock market value prediction using neural networks", In: Proc. of International Conference on Computer Information Systems and Industrial Management Applications (CISIM), Krakow, Poland, pp. 132-136, 2010.

[32] H. D. Huynh, L. M. Dang, and D. Duong, "A New Model for Stock Price Movements Prediction Using Deep Neural Network", In: Proc. of the Eighth International Symposium on Information and Communication Technology, Nha Trang City Viet Nam, pp. 57-62, 2017.

[33] C. Y. Lai, R. C. Chen, and R. E. Caraka, "Prediction Stock Price Based on Different Index Factors Using LSTM", In: Proc. of International Conference on Machine Learning and Cybernetics (ICMLC), Kobe, Japan, pp. 16, 2019. 
[34] W. Bao, J. Yue, and Y. Rao, “A deep learning framework for financial time series using stacked autoencoders and long-short term memory", PLoS One, Vol. 12, No. 7, p. e0180944, 2017.

[35] C. Stoean, W. Paja, R. Stoean, and A. Sandita, "Deep architectures for long-term stock price prediction with a heuristic-based strategy for trading simulations", PLoS One, Vol. 14, No. 10, p. e0223593, 2019.

[36] T. Kim and H. Y. Kim, "Forecasting stock prices with a feature fusion LSTM-CNN model using different representations of the same data", PLoS One, Vol. 14, No. 2, p. e0212320, 2019.

[37] M. Wen, P. Li, L. Zhang, and Y. Chen, "Stock market trend prediction using high-order information of time series", IEEE Access, Vol. 7, pp. 28299-28308, 2019.

[38] A. W. Li and G. S. Bastos, "Stock Market Forecasting Using Deep Learning and Technical Analysis: A Systematic Review", IEEE Access, Vol. 8, pp. 185232-185242, 2020.

[39] D. L. Minh, A. S. Niaraki, H. D. Huy, K. Min, and $\mathrm{H}$. Moon, "Deep learning approach for short-term stock trends prediction based on two-stream gated recurrent unit network", IEEE Access, Vol. 6, pp. 55392-55404, 2018.

[40] A. S. Saud, S. Shakya, and A. Ghosh, "Hillclimbing powered gated recurrent unit network to predict stock trading signals using the mean average convergence divergence indicator", $J$. Theor. Appl. Informtion Technol., Vol. 99, No. 7, pp. 1493-1504, 2021.

[41] Nepal Stock Exchange Ltd. [Online]. Available:http://nepalstock.com.np/ stockWisePrices. [Accessed: 02-Jul-2021].

[42] S\&P BSE SENSEX Stock Prices, [Online]. Available: https://www.bseindia.com/ markets/ equity/EQReports/StockPrcHistori.asx.

[Accessed: 02-Jul-2021].

[43] Yahoo Finance. [Online]. Available: https:// finance.yahoo.com/. [Accessed: 02-Jul-2020].

[44] A. S. Saud and S. Shakya, "Analysis of look back period for stock price prediction with RNN variants: A case study on banking sector of NEPSE", Procedia Comput. Sci., Vol. 167, pp. 788-798, 2020.

[45] Z. Yang, K. Hao, X. Cai, L. Chen, and L. Ren, "Prediction of Stock Trading Signal Based on Multi-indicator Channel Convolutional Neural Networks", In: Proc. of IEEE $8^{\text {th }}$ Data Driven Control and Learning Systems Conference (DDCLS), Dali, China, pp. 912-917, 2018.
[46] P. Kroha and M. Friedrich, "Comparison of genetic algorithms for trading strategies", Lecture Notes in Computer Science, Vol. 8327 LNCS, pp. 383-394, 2014.

[47] Y. Song, J. W. Lee, and J. Lee, "A study on novel filtering and relationship between inputfeatures and target-vectors in a deep learning model for stock price prediction", Appl. Intell., Vol. 49, No. 3, pp. 897-911, 2019.

[48] T. Matsubura, R. Akita, and K. Uehara, "Stock Price Prediction by Deep Neural Generative Model of News Articles", IEICE Trans. Inf. Syst., Vol. E101.D, No. 4, pp. 901-908, 2018.

[49] M. R. Vargas, C. E. M. D. Anjos, G. L. G. Bichara, and A. G. Evsukoff, "Deep Leaming for Stock Market Prediction Using Technical Indicators and Financial News Articles", In: Proc. of International Joint Conference on Neural Networks, Rio de Janeiro, Brazil, pp. 18, 2018.

[50] X. Tan, S. Li, C. Wang, and S. Wang, "Enhancing High Frequency Technical Indicators Forecasting Using Shrinking Deep Neural Networks", In: Proc. of $6^{\text {th }}$ International Conference on Information Management, London, UK, pp. 249-256, 2020.

[51] O. B. Sezer and A. M. Ozbayoglu, "Algorithmic financial trading with deep convolutional neural networks: Time series to image conversion approach", Appl. Soft Comput., Vol. 70, pp. 525-538, 2018.

[52] P. Oncharoen and P. Vateekul, "Deep Learning Using Risk-Reward Function for Stock Market Prediction", In: Proc. of the $2^{\text {nd }}$ International Conference on Computer Science and Artificial Intelligence, Shenzhen China, pp. 556-561. 2018. 